International Journal of Biomedicine | June 2021 - Volume 11, Issue Suppl_1: Abstracts from the Third Russian International Conference "Cryo-electron microscopy 2021: achievements and prospects"

\author{
POSTER ABSTRACT PRESENTATIONS
}

SESSION TITLE: STRUCTURE OF MEMBRANE PROTEINS

DOI: 10.21103/JJBM.11.Suppl_1.P15

\title{
Abstract P-15: Cryo-Electron Microscopy Study of Dehydrogenase Complexes Interaction with Oxidative Phosphorylation System Supercomplex
}

$\underline{\text { Konstantin Plokhikh }}^{1}$, Roman Kamyshinsky $^{1}$, Yury Chesnokov ${ }^{1}$, Semen Nesterov $^{1,2}$, Raif Vasilov ${ }^{1,3}$, Lev Yaguzhinsky ${ }^{2,3,4}$

${ }^{1}$ National Research Center "Kurchatov Institute," Moscow, Russia

${ }^{2}$ Moscow Institute of Physics and Technology, Dolgoprudny, Russia ${ }^{3}$ Belozersky Research Institute for Physico-Chemical Biology, Moscow, Russia ${ }^{4}$ Institute of Cytochemistry and Molecular Pharmacology, Moscow, Russia

Background: Electron transport chain (ETC) complexes, pyruvate dehydrogenase complex (PDC), and $\alpha$-ketoglutarate dehydrogenase complex (KGDC) are important elements in mitochondrial metabolism. The localization of the aforementioned protein complexes differs since oxidative phosphorylation complexes are membrane proteins, while dehydrogenase complexes (DCs) are contained in the mitochondrial matrix. Our previous cryo-electron tomography (cryo-ET) studies showed the existence of a full oxidative phosphorylation system supercomplex consisting of ETC complexes and ATP synthases (Nesterov et al., 2021). Literature data also shows the binding of fatty acid oxidation enzymes to ETC complex I (Wang et al., 2010). Although it has long been shown that PDCs can bind to complex I (Sumegi et al., 1984) in vitro, this has not been visualized directly in mitochondria and the binding mechanisms are still unknown.

Methods: The mitochondria were isolated from Wistar rat heart ventricles according to a standard procedure (Nesterov et al., 2021). The dense mitochondrial suspension was diluted to $\sim 0.3 \mathrm{mg} / \mathrm{ml}$ in a respiration medium. Phosphorylation was started 10 minutes prior to vitrification. Experimental data was obtained by cryo-ET using Titan Krios and processed with IMOD and RELION.

Results: The tomograms show that the significant part of DCs is localized near the inner membrane of partially destroyed mitochondria in an array-like fashion. 
Sole PDCs and KGDCs can be identified on the images and their position appears to be close to ETC complex I. Subtomogram averaging of close to the membrane DCs showed that there is no specific density between them, suggesting that they are not linked with identical proteins or that this link may be soft. Significant damage to the mitochondrial membrane leads to the formation of membrane-unbound DCs fraction. It suggests that coupling of DCs with ETC complexes can be controlled in vivo by the topology of the inner mitochondrial membrane and the volume of the mitochondrial matrix.

Conclusion: The obtained results show a possibility of unprecedentedly large multienzyme complex formation, including almost all main mitochondrial metabolic systems. Although cryo-ET of partially destroyed mitochondria showed close localization of PDC and KGDC to complex I, further studies are required in intact mitochondria. The mechanism of their binding also remains an open question.

Key Words: cryo-ET $\bullet$ pyruvate dehydrogenase complex $\bullet \alpha$-ketoglutarate dehydrogenase complex $\bullet$ oxidative phosphorylation

This work was supported by the Russian Foundation for Basic Research (Grant No. 19-04-00835) and NRC "Kurchatov Institute" (thematic plan "Study of the processes of generation, transmission and distribution of energy in living organisms")

*Corresponding author: Konstantin Plokhikh.E-mail: konstantin.plokhikh@phystech.edu

International Journal of Biomedicine. 2021;11 Suppl 1: S17-18.

doi: 10.21103/IJBM.11.Suppl_1.P15

(C)2021 International Medical Research and Development Corporation 